Published in final edited form as:

Sex Transm Dis. 2012 October ; 39(10): 807-812. doi:10.1097/OLQ.0b013e3182631c79.

\title{
Association between Trichomonas vaginalis and vaginal bacterial community composition among reproductive-age women
}

Rebecca M. Brotman, PhD, MPH ${ }^{1,2}$, L. Latey Bradford, BS ${ }^{1}$, Melissa Conrad, M.Sc. ${ }^{3}$, Pawel Gajer, PhD $^{1}$, Kevin Ault, MD ${ }^{4,5}$, Ligia Peralta, MD $^{6}$, Larry J. Forney, PhD $^{7,8}$, Jane M. Carlton, Ph.D. ${ }^{3}$, Zaid Abdo, PhD ${ }^{8,9,10}$, and Jacques Ravel, PhD ${ }^{1,11}$

${ }^{1}$ Institute for Genome Sciences, University of Maryland School of Medicine, Baltimore, MD

${ }^{2}$ Department of Epidemiology and Public Health, University of Maryland School of Medicine, Baltimore, MD

${ }^{3}$ Center for Genomics and Systems Biology, Department of Biology, New York University, New York, NY

${ }^{4}$ Department of Gynecology and Obstetrics and the Emory Vaccine Center, Emory University, Atlanta, GA

${ }^{5}$ Hubert Department of Global Health, Rollins School of Public Health, Emory University Atlanta, GA

${ }^{6}$ Department of Pediatrics, Adolescent and Young Adult Medicine, University of Mary land School of Medicine, Baltimore, MD

${ }^{7}$ Department of Biological Sciences, University of Idaho, Moscow, ID

${ }^{8}$ Institute for Bioinformatics and Evolutionary Studies (IBEST), University of Idaho, Moscow, ID ${ }^{9}$ Department of Mathematics, University of Idaho, Moscow, ID

${ }^{10}$ Department of Statistics, University of Idaho, Moscow, ID

${ }^{11}$ Department of Microbiology and Immunology University of Maryland School of Medicine, Baltimore, MD

\section{Abstract}

Objectives-Some vaginal bacterial communities are thought to prevent infection by sexually transmitted organisms. Prior work demonstrated that the vaginal microbiota of reproductive-age women cluster into five types of bacterial communities; 4 dominated by Lactobacillus species ( $L$. iners, L. crispatus, L. gasseri, L. jensenii), and one (termed community state type (CST) IV) lacking significant numbers of lactobacilli and characterized by higher proportions of Atopobium,

Address for reprints and corresponding author: Rebecca M. Brotman, PhD, MPH, Assistant Professor, Department of Epidemiology and Public Health, Institute for Genome Sciences, University of Maryland School of Medicine, 801 West Baltimore Street, Room Number 633, Baltimore, MD 21201, phone: (410) 706-6767; fax: (410) 706-1482, rbrotman@ som.umaryland.edu. Jacques Ravel, $\mathrm{PhD}$, Associate Professor, Department of Microbiology and Immunology, Institute for Genome Sciences, University of Maryland School of Medicine, 801 West Baltimore Street, Room 611, Baltimore, MD 21201, phone: (410) 706-5674; fax: (410) 706-1482, jravel@som.umaryland.edu.

Presentation information: Presented in part as a poster at the International Human Microbiome Congress, March 9-11, 2011,

Vancouver Canada and as an oral presentation at the International Society for Sexually Transmitted Disease Research, 19th Biennial Congress, July 11-13, 2011, Quebec City.

Disclosure: The authors report no conflict of interest. 
Prevotella, Parvimonas, Sneathia, Gardnerella, Mobiluncus, and other taxa. We sought to evaluate the relationship between vaginal bacterial composition and Trichomonas vaginalis.

Methods-Self-collected vaginal swabs were obtained cross-sectionally from 394 women equally representing four ethnic/racial groups. $T$. vaginalis screening was performed using PCR targeting the $18 \mathrm{~S}$ rRNA and $\beta$-tubulin genes. Vaginal bacterial composition was characterized by pyrosequencing of barcoded 16S rRNA genes. A panel of eleven microsatellite markers was used to genotype $T$. vaginalis. The association between vaginal microbiota and $T$. vaginalis was evaluated by exact logistic regression.

Results- $T$. vaginalis was detected in $2.8 \%$ of participants (11/394). Of the eleven $T$. vaginalispositive cases, eight (72\%) were categorized as CST-IV, two (18\%) as communities dominated by L. iners and one (9\%) as L. crispatus-dominated (p-value:0.05). CST-IV microbiota were associated with an 8-fold increased odds of detecting $T$. vaginalis compared to women in the $L$. crispatus-dominated state (OR:8.26, 95\% CI:1.07-372.65). Seven of the 11 T. vaginalis isolates were assigned to two genotypes.

Conclusion-T. vaginalis was associated with vaginal microbiota consisting of low proportions of lactobacilli and high proportions of Mycoplasma, Parvimonas, Sneathia, and other anaerobes.

\section{Keywords}

Trichomonas vaginalis; vaginal microbiota

\section{Introduction}

The National Health and Nutrition Examination Survey found in 2001-2004 that 3.1\% of U.S. women were positive for Trichomonas vaginalis genital infections (1), making it a more pervasive sexually transmitted infection (STI) than Neisseria gonorrhea and Chlamydia trachomatis combined (2). In 1999, Cates et al. estimated an incidence of five million new cases of trichomoniasis annually in the U.S.(3) Racial disparity in trichomoniasis rates are particularly striking with non-Hispanic black women having over ten-fold higher prevalence rates (13.3\%) than non-Hispanic white (1.3\%) or Mexican American (1.8\%) women.(1) In a longitudinal study of 268 adolescents in Indianapolis, Indiana, incident cases of $T$. vaginalis were detected in $23 \%$ of participants and re-infection episodes occurred in $31 \%$ of those individuals.(4) Although trichomoniasis is a treatable STI (5), it continues to represent a critical public health issue as over $85 \%$ of cases are asymptomatic (1), and the protozoan is associated with numerous adverse outcomes, including preterm delivery of low-birth weight infants (6) and increased susceptibility to and transmission of HIV infection. $(7 ; 8)$ It has been estimated that 746 new HIV cases among U.S. women each year can be attributed to enhanced acquisition of HIV by underlying $T$. vaginalis infection.(9)

Lactic acid producing bacteria in the vagina, made up in large part by Lactobacillus sp., are thought to play a key protective role in preventing urogenital infection $(10 ; 11)$ by lowering the vaginal $\mathrm{pH}$, generating bacteriostatic and bacteriocidal compounds, (12) and through possible competitive exclusion of non-indigenous organisms that include opportunistic and overt pathogens. Ravel et al. (13) recently demonstrated by high-throughput cultureindependent molecular analysis that the vaginal bacterial communities of North American women can be classified into five community state types (CST); four of which are dominated by one of Lactobacillus iners, L. crispatus, L. gasseri or L. jensenii. The remaining group (termed CST-IV) comprised $27 \%$ of the women in the study and was characterized by higher vaginal $\mathrm{pH}$ and greater relative abundance of strict anaerobic organisms, including Atopobium, Prevotella, Dialister, Gardnerella, Megasphaera, 
Peptoniphilus, Sneathia, Eggerthella, Aerococcus, Finegoldia, and Mobiluncus. Our overall hypothesis is that the five different vaginal bacterial communities differ in terms of their ability to fend off colonization by pathogens.

In the current study, we sought to compare the vaginal microbiota of $T$. vaginalis-positive and $T$. vaginalis-negative women using cultivation-independent methods based on the analysis of $16 \mathrm{~S}$ rRNA genes. This molecular epidemiologic study, using high-throughput cultivation-independent methodologies, is able for the first time to decipher how vaginal bacterial communities differ among $T$. vaginalis cases. Prior work on the association of vaginal microbiota and STIs have focused on cultivation-based studies of lactobacilli and microscopy evaluation of Gram stained smears.(14-18) Cultivation-based approaches are hindered as many microbial species resist cultivation in the laboratory (19) and Gram stain provides only morphological information (20). In addition, we genotyped the T. vaginalispositive samples using species-specific microsatellite markers, and successfully assigned seven of the eleven isolates to the two types that characterize the parasite's global population structure (21).

\section{Materials and Methods}

\section{Clinical study design}

This report utilized secondary analysis and repository samples from the study by Ravel et al. of 394 North American women representing four ethnic/racial groups (white, black, Hispanic and Asian) which has been previously described.(13) Briefly, from 2008-2009, non-pregnant, women aged 12 to 45 with regular menstrual cycles were recruited to an observational, cross-sectional study in one of two U.S. cities (Atlanta or Baltimore). Women were ineligible if they reported vaginal discharge in the prior 48 hours, pregnancy, use of intravaginal products or sexual activity in the prior 48 hours, use of antibiotics or antimycotics in the prior 30 days, significant medical or gynecological conditions (eg. posthysterectomy or immunocompromised status such as congenital, acquired or secondary to medication), or current menstrual bleeding or vaginal symptoms. Participants underwent a detailed interview in a private office, which was self-administered or administered by a female interviewer. The interview was focused on personal hygiene, sexual and contraceptive history and demographic information. Participants self-reported racial classification using pre-defined categories. Data on self-reported racial identification was collected because of observed disparities in STI rates between U.S. racial groups (1).

Using validated protocols $(22 ; 23)$, participants self-collected two mid-vaginal swabs using the Elution-swab system (Copan) that were respectively used to prepare a smear for Nugent Gram stain scoring (20) and to characterize the composition and structure of the resident bacterial communities using pyrosequencing of barcoded 16S rRNA genes. (13) The Nugent scoring criteria reflects the relative abundance of large Gram-positive rods (lactobacilli), Gram-negative and Gram-variable rods and cocci (i.e., Gardnerella vaginalis, Prevotella, Porphyromonas, and peptostreptococci), and curved Gram-negative rods (Mobiluncus).(20) This technique assesses the relative numbers of morphotypes, allowing a coarse classification of certain vaginal microbes. Nugent scores reflect the range of vaginal microbiota states. A score of $0-3$ is designated as normal, 4-6 as an intermediate state and 7-10 as a high Nugent score indicative of bacterial vaginosis (BV) diagnosis. (20) Participants also self-collected and reported vaginal $\mathrm{pH}$ using the $\mathrm{VpH}$ glove (Inverness Medical). Study staff confirmed the $\mathrm{pH}$ measurement and also took a digital photo of the $\mathrm{pH}$ strip adjacent to the color scale.

The study was approved by Institutional Review Boards at the University of Maryland School of Medicine and the Emory University School of Medicine. All participants provided 
written informed consent. The study was registered at clinicaltrials.gov under ID NCT00576797.

\section{T. vaginalis screening and primers}

Whole genomic DNA extraction was performed on the self-collected vaginal swabs as previously described.(13) $T$. vaginalis was detected by polymerase chain reaction (PCR)based methods using two primer sets, TV16Sf-2/TV16Sr-2 (a $323 \mathrm{bp}$ amplicon) targeting the 18S rRNA gene (24) and TVK3/TVK7 (a 300 bp amplicon) targeting a DNA repeat region of the $T$. vaginalis genome. (25) A third primer set, BTub3/bkmt (a $195 \mathrm{bp}$ amplicon), targeting the $\beta$-tubulin gene was only used on four discordant samples and confirmed the absence of $T$. vaginalis.(24) All PCRs were performed as previously reported and amplicons were visualized by gel electrophoresis.

\section{T. vaginalis genotyping}

T. vaginalis-positive samples were genotyped using eleven microsatellite (MS) loci as previously described.(26) This subset of the published 21 MS panel consists of the most informative set of markers that allow distinction between type 1 and type 2 parasites, the two groups that $T$. vaginalis parasites are found to cluster into world-wide (21), and is ideal for use with clinical samples where low levels of target parasite DNA are a confounding factor. All genotyping PCR reactions were performed in duplicate and size polymorphisms were measured by capillary gel electrophoresis on an ABI 3130xl sequencer. GeneMapper 4.O (ABI, Foster City, CA) was used to score MS allele sizes, and all peaks were manually edited. Discrepant allele calls were repeated for a third time. Type assignment was then performed using the Bayesian clustering program, STRUCTURE 2.2.(27)

\section{Statistical analysis}

Fisher's exact test and exact logistic regression were used to determine the association between vaginal bacterial CST (13) and the detection of $T$. vaginalis. Factors collected from questionnaires that had been identified on the basis of previous literature and biologic plausibility were evaluated in univariable analyses. The limited number of observed $T$. vaginalis-positive cases (11 women) would not allow for further multivariable odds ratio analyses. To assess differences in the bacterial community structure between $T$. vaginalis cases and controls, we also utilized distance based redundancy analysis (dRDA) (28) and a permutation test to assess significance of the results. Data were analyzed using STATA/SE 10.0 for Windows (Stata Corporation, College Station, Texas) and the dRDA was performed using the Vegan package implemented in R (R Foundation for Statistical Computing, Vienna, Austria) (29;30).

\section{Results}

Of 394 women, eleven $(2.78 \%)$ were positive for $T$. vaginalis. Among the $T$. vaginalispositive cases, eight (72\%) were detected among women belonging to the low-lactobacilli CST-IV group, while two (18\%) and one (9\%) were among women with bacterial communities dominated by $L$. iners and $L$. crispatus respectively (p-value: 0.05 , Table 1 ), reflecting the differences in classification of bacterial community state type by $T$. vaginalis positivity. Figure 1 displays the proportions of bacterial taxa found among the $383 T$. vaginalis-negative cases in comparison to the $11 \mathrm{~T}$. vaginalis-positive study participants.

A MANOVA table resulting from dRDA analysis (data not shown) indicated that there were statistically significant differences in bacterial community structure within CST-IV between the $T$. vaginalis-positive and $T$. vaginalis-negative samples (permuted $\mathrm{p}$-value $=0.013$, number of permutations $=10000$ ). Among women assigned to CST-IV, Mycoplasma and, to 
a lesser degree, Parvimonus and Sneathia were associated with $T$. vaginalis-positivity and Streptococcus, Prevotella, L. iners and Atopobium were associated, although not as strongly, with $T$. vaginalis-negative controls. However, only $2.5 \%$ of the total variation between the communities of CST-IV is explained by $T$. vaginalis, indicating that $T$. vaginalis is not a robust predictor of community structure in this study or there were too few cases observed. For detailed information on the composition of vaginal communities of the $T$. vaginalispositive cases, see supplementary information in Ravel et al. (13) for the following double blinded identification numbers-- S023, S070, S102, S103, S104, S109, S234, S255, S307, S329, S383.

Of the eleven T. vaginalis cases, ten (91\%) were observed among African-American women, one from an Asian woman (9\%) and none among the Hispanic or Caucasian women (Table 1, differences between case and control, p-value: 0.00002). In addition, none of the T. vaginalis-positive women reported hormonal contraceptive use versus $28 \%$ of controls (pvalue: 0.04$)$. The proportions in the paired levels of vaginal $\mathrm{pH}$ were also significantly different (p-value: 0.01), with $\mathrm{pH}$ higher (over 4.5) in $90 \%$ of cases compared to $46 \%$ of $T$. vaginalis-negative women. ( $\mathrm{pH}$ is also displayed in Figure 1.)

In exact logistic regression modeling, CST-IV was associated with a significant 8-fold increased odds for detection of $T$. vaginalis compared to women in the L. crispatus community state type (odds ratio (OR): $8.26,95 \%$ CI: 1.07-372.65). (Table 2) Similarly, high Nugent score was associated with a 9-fold increased odds for the presence of $T$. vaginalis compared to women with low Nugent scores (OR: 9.53, 95\% CI: 1.77-95.71).

Seven of the eleven $T$. vaginalis-positive samples were successfully genotyped at six or more microsatellite loci. Three isolates were found to cluster within type 1 (S023, S102, S103), and four isolates within type 2 (S070, S104, S234, and S329), and none of the seven isolates appeared to be made up of more than one genotype. No statistically significant associations between isolate type and participant characteristics were found, including vaginal bacterial community state type, $\mathrm{pH}$, Nugent Gram stain score, age, race/ethnicity, use of hormonal contraception or number of sexual partners (data not shown).

\section{Comment}

Our study revealed a disproportionate burden of $T$. vaginalis among women whose vaginal bacterial communities were determined by molecular analysis to be comprised of higher proportion of members of the genera Mycoplasma, Parvimonas, Sneathia, and other taxa (Figure 1). These samples concomitantly displayed relatively low abundance of lactobacilli. A limitation of our study is that we can only report on the association between vaginal bacterial communities and the presence of $T$. vaginalis infection; we cannot establish in this cross-sectional study design whether the observed vaginal community state type occurred before or after $T$. vaginalis acquisition. However, we can hypothesize that a highly plausible mechanism for a causal relationship is the lack of production of lactic acid by lactobacilli. O'Hanlon et al, demonstrated inhibition of bacteria by lactic acid and we can hypothesize that lactic acid may play a protective role against $T$. vaginalis as well.(11) Prior longitudinal studies have shown a significant association between vaginal microbiota (as determined by Amsel's clinical criteria for BV or Gram stain analysis) and T. vaginalis acquisition. $(15 ; 16 ; 31)$ Our data provides the critical impetus that a large, longitudinal study with frequent sampling is needed to determine if certain types of vaginal bacterial communities predispose women to acquisition of STIs or if the observed community state types are the result of the STI. Clinical intervention on the former could reduce risk for STI acquisition.

In this report, we also sought to detect whether specific correlations between the vaginal microbiota and $T$. vaginalis genotype could be made; however, with the low number of $T$. 
vaginalis positive samples genotyped, we failed to find any significant associations. It has been demonstrated that globally $T$. vaginalis clusters into two genetically distinct types, type 1 and type 2, which appear to be equally geographically widespread.(21) Future studies with larger sample sizes should be considered.

Our finding of an association between $T$. vaginalis and CST-IV may be due to confounding based on two observations. First, African American women have higher prevalence rates for T. vaginalis (1); and second, CST-IV is more commonly observed among African-American women (13). Ravel et al. previously reported that African American women made up 39\% of women in CST-IV,(13) and among the T. vaginalis-positive cases in our study, 90\% were African American women. However, it is also possible that the racial disparity in $T$. vaginalis observed in our study might reflect a STD transmission core. Laumann et al. modeled bacterial STD transmission in a nationally representative dataset and demonstrated that African Americans' higher STI rates could be partially explained by the patterns of sexual networks and segregated partner choices (assortative mating). (32) The latter would suggest that the higher $T$. vaginalis prevalence rate among African American women in the current study indicates that women with communities with relatively low-lactobacilli abundance (CST-IV) could be at increased risk for $T$. vaginalis acquisition upon exposure.

Strengths of this study include a large, racially diverse study population, validated protocols for self-sampling $(22 ; 23)$ and the use of molecular techniques to detect both $T$. vaginalis and vaginal bacterial community state types. Published PCR sensitivities for $T$. vaginalis range from $81 \%-97 \%$, exceeding the sensitivities which have been reported for wet mount evaluation, Papanicolaou test, DNA probe, or culture. (5)

Limitations of the study include the recruitment of only women who reported no vaginal discharge and the limited sample size of $11 \mathrm{~T}$. vaginalis cases out of a study of 394 women. Although statistically significant, the odds ratio estimate for the low-lactobacillus CST-IV should be taken cautiously owing to the wide confidence intervals likely due to the small number of observed cases. There were no $T$. vaginalis cases detected among the $L$. gasseri and $L$. jensenii community state types which may reflect protection by these Lactobacillus spp. or limitations of the sample size. In addition, a limitation of PCR is that viable and nonviable organisms cannot be distinguished. Participants reported they had not taken an antibiotic in the prior 30 days and one of the $T$. vaginalis-positive cases indicated on survey questioning that she had a $T$. vaginalis infection treated in the prior 60 days. Previously treated infections could potentially have resulted in a positive PCR result without viable organisms although dead cells and free DNA are unlikely to persist.

It is now recognized that the vaginal microbiota play a major role in maintaining the reproductive health of women. A recent study by Gatski et al. reported that high Nugent Gram stain score was associated with early failure of metronidazole single dose treatment for trichomoniasis among HIV-positive women.(33) In addition, differences in vaginal bacterial community composition constitute a significant variable that has not been emphasized in current research on risk factors for STI acquisition. Future research should focus on functional differences of the various vaginal bacterial communities, including the dominant lactobacilli species (and strains), and their ability to protect against urogenital pathogens. Longitudinal studies of vaginal microbiome and STIs are critical in order to determine temporality and causality. All women recruited to the current study did not report vaginal discharge, highlighting further that $T$. vaginalis is often an asymptomatic infection and suggests that screening could increase awareness, reduce transmission. Rapid screening tests for $T$. vaginalis (5) are available to medical practitioners and self-collected vaginal swabs can be utilized for $T$. vaginalis detection (34). In addition, use of self-collected midvaginal swabs for microbiome analysis has been validated $(22 ; 23)$ and provides feasibility 
for field-based longitudinal studies of STI incidence (16). An increased understanding of the vaginal microbial ecosystem could lead to more effective and personalized strategies for the prevention of genital $T$. vaginalis and other STIs.

\section{Acknowledgments}

Sources of financial support, including granting agency and grant number: This study was supported by National Institutes of Health grants U01-AI070921 (Ravel), K01-AI080974 (Brotman), UH2-AI083264 (Ravel and Forney) and R21AI083954 (Carlton). The study was also supported in part by NIH grants UL1-RR025008, KL2-RR025009 and TL1-RR025010 (Ault).

We thank Bishoy Michael, Hongqui Yang, Joyce Sakamoto, Grace Maldarelli, Sergio Mojica, Stacey McCulle and Sara Koenig (all at University of Maryland, Institute for Genome Sciences) for technical help, as well as Dr. Charlotte Gaydos (Johns Hopkins School of Medicine) for providing the DNA of Trichomonas vaginalis that was used as positive control in the PCR assays. We would also like to acknowledge Dr. Khalil Ghanem (Johns Hopkins School of Medicine) for insightful comments in the review of the manuscript.

\section{References}

1. Sutton M, Sternberg M, Koumans EH, et al. The prevalence of Trichomonas vaginalis infection among reproductive-age women in the United States, 2001-2004. Clin Infect Dis. 2007; 45:1319_ 1326. [PubMed: 17968828]

2. Datta SD, Sternberg M, Johnson RE, et al. Gonorrhea and chlamydia in the United States among persons 14 to 39 years of age, 1999 to 2002. Ann Intern Med. 2007; 147:89-96. [PubMed: 17638719]

3. Cates W Jr. Estimates of the incidence and prevalence of sexually transmitted diseases in the United States. American Social Health Association Panel. Sex Transm Dis. 1999; 26:S2-S7. [PubMed: 10227693]

4. Van Der PB, Williams JA, Orr DP, et al. Prevalence, incidence, natural history, and response to treatment of Trichomonas vaginalis infection among adolescent women. J Infect Dis. 2005; 192:2039-2044. [PubMed: 16288365]

5. Workowski KA, Berman S. Centers for Disease Control and Prevention (CDC). Sexually transmitted diseases treatment guidelines, 2010. MMWR Recomm Rep. 2010; 59:1-110. [PubMed: 21160459]

6. Cotch MF, Pastorek JG, Nugent RP, et al. Trichomonas vaginalis associated with low birth weight and preterm delivery. The Vaginal Infections and Prematurity Study Group. Sex Transm Dis. 1997; 24:353-360. [PubMed: 9243743]

7. Thurman A, Doncel GF. Innate Immunity and Inflammatory Response to Trichomonas vaginalis and Bacterial Vaginosis: Relationship to HIV Acquisition. American Journal of Reproductive Immunology. 2010 no.

8. Kissinger P, Amedee A, Clark RA, et al. Trichomonas vaginalis treatment reduces vaginal HIV-1 shedding. Sex Transm Dis. 2009; 36:11-16. [PubMed: 19008776]

9. Chesson HW, Blandford JM, Pinkerton SD. Estimates of the annual number and cost of new HIV infections among women attributable to trichomoniasis in the United States. Sex Transm Dis. 2004; 31:547-551. [PubMed: 15480116]

10. Lai SK, Hida K, Shukair S, et al. Human immunodeficiency virus type 1 is trapped by acidic but not by neutralized human cervicovaginal mucus. J Virol. 2009; 83:11196-11200. [PubMed: 19692470]

11. O'Hanlon DE, Moench TR, Cone RA. In vaginal fluid, bacteria associated with bacterial vaginosis can be suppressed with lactic acid but not hydrogen peroxide. BMC Infect Dis. 2011; 11:200. [PubMed: 21771337]

12. Hillier, SL.; Holmes, KK.; Marrazzo, JM. Sexually Transmitted Diseases. New York: McGrawHill, Health Professions Division; 2008. Bacterial Vaginosis; p. 737-768.

13. Ravel J, Gajer P, Abdo Z, et al. Vaginal microbiome of reproductive-age women. Proc Natl Acad Sci U S A. 2011; 108 (Suppl 1):4680-4687. [PubMed: 20534435] 
14. Cherpes TL, Meyn LA, Krohn MA, et al. Association between acquisition of herpes simplex virus type 2 in women and bacterial vaginosis. Clin Infect Dis. 2003; 37:319-325. [PubMed: 12884154]

15. Martin HL, Richardson BA, Nyange PM, et al. Vaginal lactobacilli, microbial flora, and risk of human immunodeficiency virus type 1 and sexually transmitted disease acquisition. J Infect Dis. 1999; 180:1863-1868. [PubMed: 10558942]

16. Brotman RM, Klebanoff MA, Nansel TR, et al. Bacterial vaginosis assessed by gram stain and diminished colonization resistance to incident gonococcal, chlamydial, and trichomonal genital infection. J Infect Dis. 2010; 202:1907-1915. [PubMed: 21067371]

17. Moodley P, Connolly C, Sturm AW. Interrelationships among human immunodeficiency virus type 1 infection, bacterial vaginosis, trichomoniasis, and the presence of yeasts. J Infect Dis. 2002; 185:69-73. [PubMed: 11756983]

18. Torok MR, Miller WC, Hobbs MM, et al. The association between Trichomonas vaginalis infection and level of vaginal lactobacilli, in nonpregnant women. J Infect Dis. 2007; 196:11021107. [PubMed: 17763335]

19. Bakken LR. Separation and Purification of Bacteria from Soil. Appl Environ Microbiol. 1985; 49:1482-1487. [PubMed: 16346816]

20. Nugent RP, Krohn MA, Hillier SL. Reliability of diagnosing bacterial vaginosis is improved by a standardized method of gram stain interpretation. J Clin Microbiol. 1991; 29:297-301. [PubMed: 1706728]

21. Conrad MD, Gorman AW, Schillinger JA, et al. Extensive Genetic Diversity, Unique Population Structure and Evidence of Genetic Exchange in the Sexually Transmitted Parasite Trichomonas vaginalis. PLoS Negl Trop Dis. 2012; 6:e1573. [PubMed: 22479659]

22. Forney LJ, Gajer P, Williams CJ, et al. Comparison of self-collected and physician-collected vaginal swabs for microbiome analysis. J Clin Microbiol. 2010; 48:1741-1748. [PubMed: 20200290]

23. Bai G, Gajer P, Nandy M, et al. Comparison of storage conditions for human vaginal microbiome studies. PLoS.ONE. 2012 Ref Type: In Press.

24. Simpson P, Higgins G, Qiao M, et al. Real-time PCRs for detection of Trichomonas vaginalis betatubulin and 18S rRNA genes in female genital specimens. J Med Microbiol. 2007; 56:772-777. [PubMed: 17510262]

25. Kengne P, Veas F, Vidal N, et al. Trichomonas vaginalis: repeated DNA target for highly sensitive and specific polymerase chain reaction diagnosis. Cell Mol Biol (Noisy -le-grand). 1994; 40:819_ 831. [PubMed: 7812190]

26. Conrad M, Zubacova Z, Dunn LA, et al. Microsatellite polymorphism in the sexually transmitted human pathogen Trichomonas vaginalis indicates a genetically diverse parasite. Mol Biochem Parasitol. 2011; 175:30-38. [PubMed: 20813140]

27. Pritchard JK, Stephens M, Donnelly P. Inference of population structure using multilocus genotype data. Genetics. 2000; 155:945-959. [PubMed: 10835412]

28. Legendre P, Anderson MJ. DISTANCE-BASED REDUNDANCY ANALYSIS: TESTING MULTISPECIES RESPONSES IN MULTIFACTORIAL ECOLOGICAL EXPERIMENTS. Ecological Monographs. 1999; 69:1-24.

29. Vegan: Community Ecology Package. [computer program]. R package version 2.0-3. 2012. http://CRAN.R-project.org/package=vegan

30. R: A language and environment for statistical computing [computer program]. Vienna, Austria: R Foundation for Statistical Computing; 2005. http://www.R-project.org

31. Peipert JF, Lapane KL, Allsworth JE, et al. Bacterial vaginosis, race, and sexually transmitted infections: does race modify the association? Sex Transm Dis. 2008; 35:363-367. [PubMed: 18360319]

32. Laumann EO, Youm Y. Racial/ethnic group differences in the prevalence of sexually transmitted diseases in the United States: a network explanation. Sex Transm Dis. 1999; 26:250-261. [PubMed: 10333277]

33. Gatski M, Martin DH, Levison J, et al. The influence of bacterial vaginosis on the response to Trichomonas vaginalis treatment among HIV-infected women. Sex Transm Infect. 2011 
34. Crucitti T, Van DE, Tehe A, et al. Comparison of culture and different PCR assays for detection of Trichomonas vaginalis in self collected vaginal swab specimens. Sex Transm Infect. 2003; 79:393-398. [PubMed: 14573835] 
A.

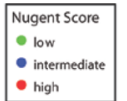

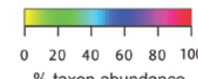

$\%$ taxon abundance

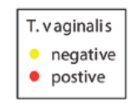

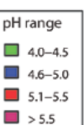

> $>5.5$

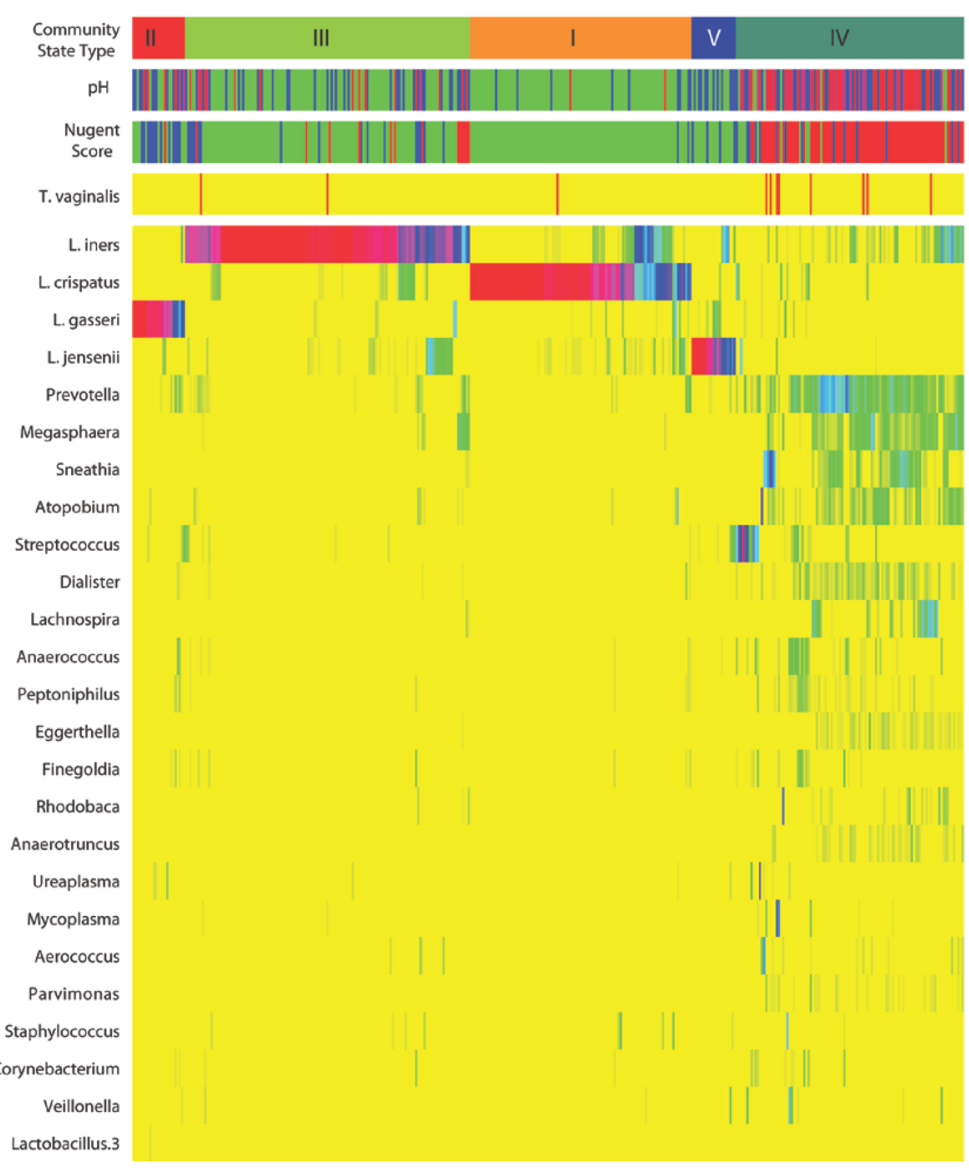

B.

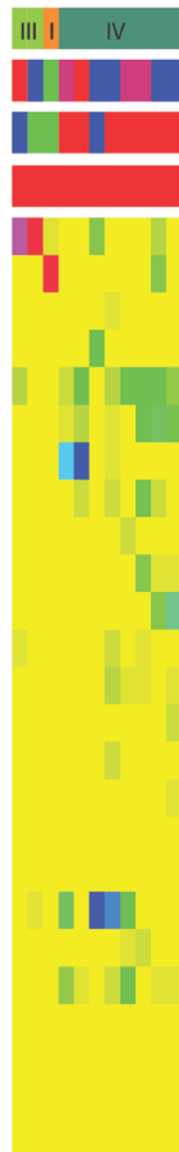

Figure 1.

Heatmap of relative percent abundance of bacterial taxa found in the vaginal bacterial communities of 394 reproductive age women (A) and of the $11 \mathrm{~T}$. vaginalis positive cases (B). Vaginal bacterial community state type is indicated as described by Ravel et al ${ }^{14}$. Nugent Gram stain scores, $\mathrm{pH}$ measurements and $T$. vaginalis positivity are shown for each of the 394 samples. 


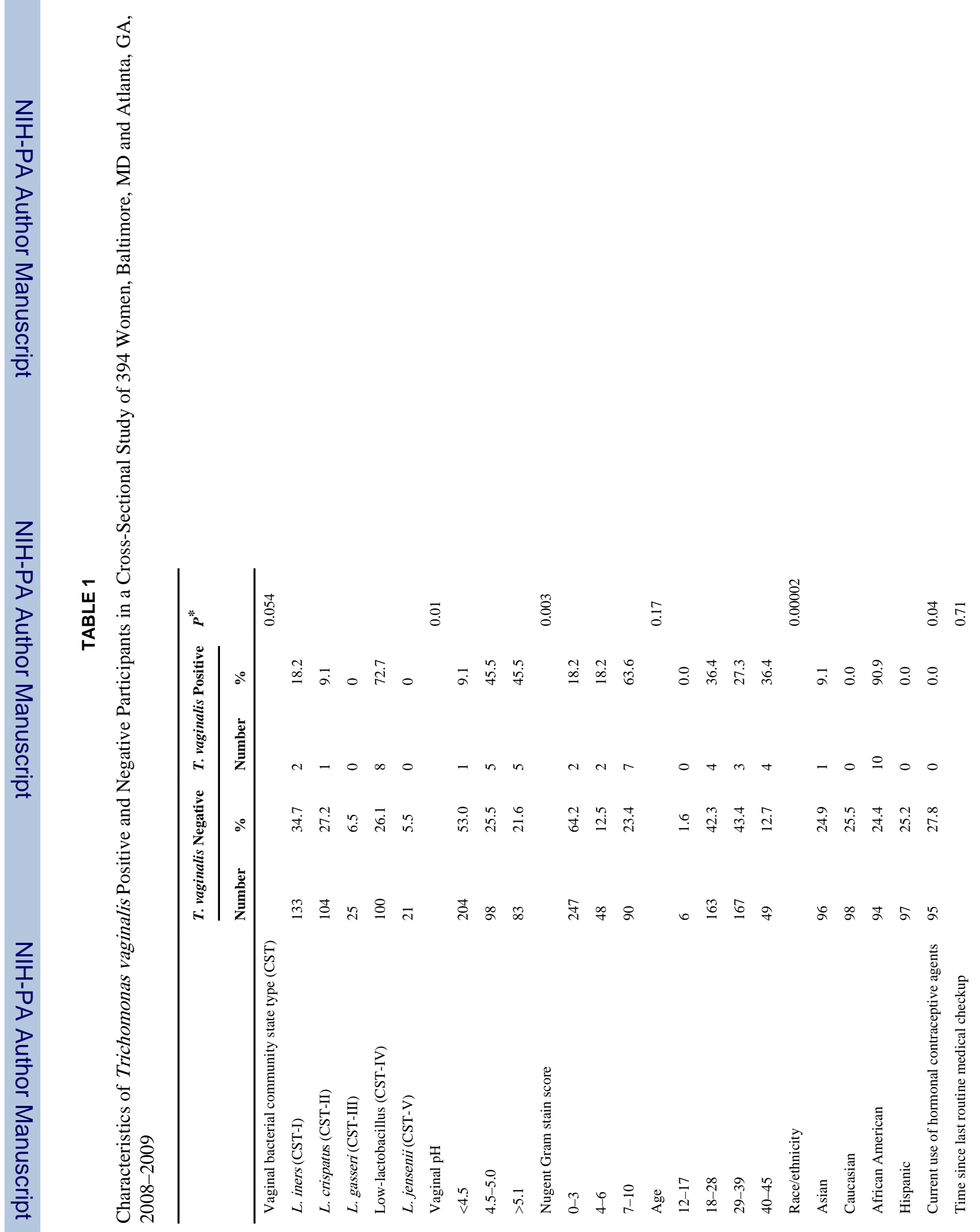

Sex Transm Dis. Author manuscript; available in PMC 2013 October 01. 


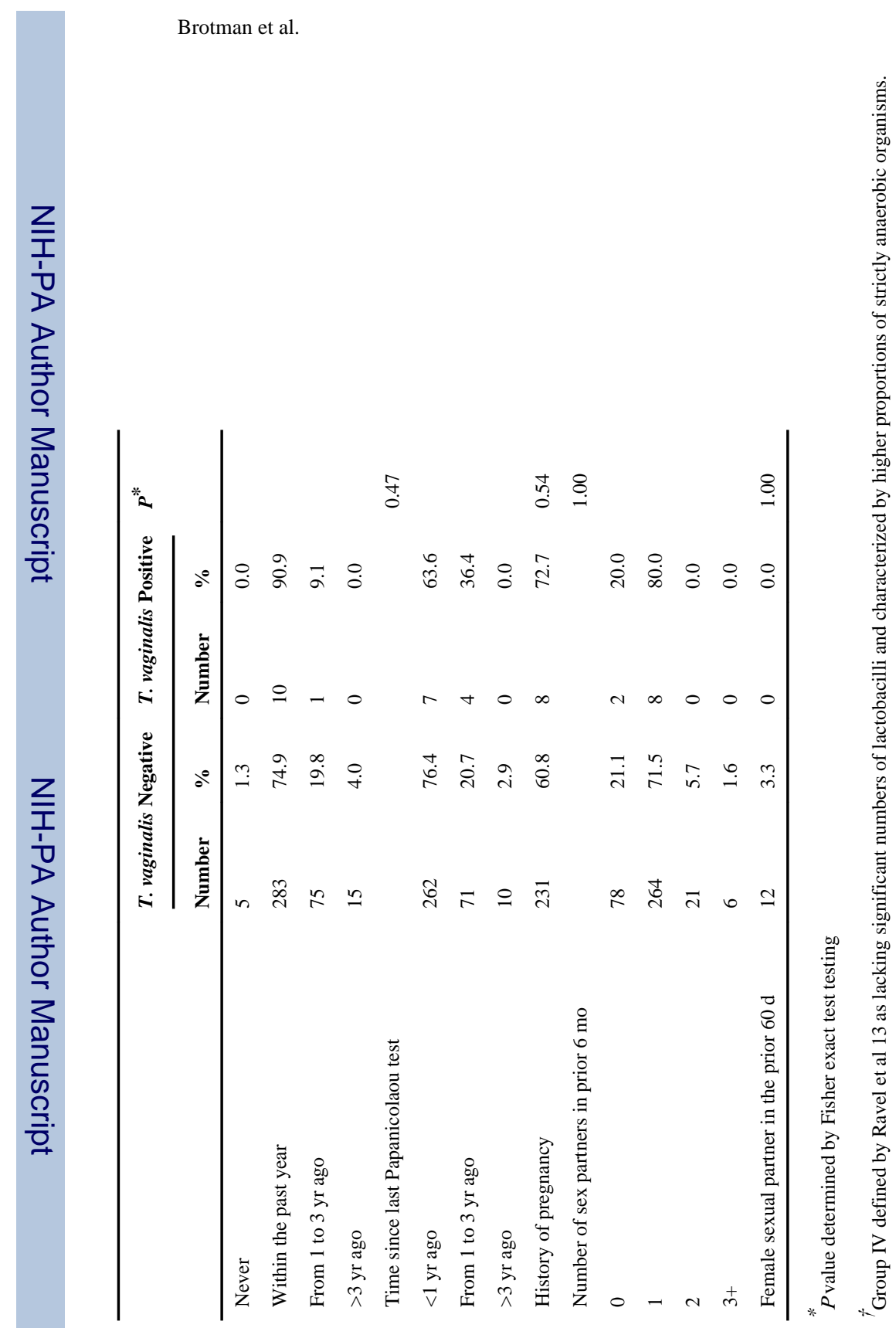

Page 12

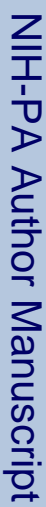

Sex Transm Dis. Author manuscript; available in PMC 2013 October 01. 
TABLE 2

Odds of Trichomonas vaginalis Detection by Various Factors, Baltimore, MD and Atlanta, GA, 2008-2009 (N $=394$ Women)

\begin{tabular}{|c|c|c|c|c|c|}
\hline Characteristic & OR ${ }^{*}$ & $\boldsymbol{P}$ & \multicolumn{2}{|c|}{$95 \% \mathrm{CI}$} & \\
\hline \multicolumn{6}{|c|}{ Vaginal bacterial community state type } \\
\hline L. crispatus & REF & - & - & - & \\
\hline L. gasseri ${ }^{\dagger}$ & - & - & - & - & \\
\hline L. iners & 1.56 & 1.00 & 0.08 & 93.14 & \\
\hline Low-lactobacillus/CST-IV $\neq$ & 8.26 & 0.04 & 1.07 & 372.65 & \\
\hline L. jensenii ${ }^{\dagger}$ & - & - & - & - & \\
\hline \multicolumn{6}{|l|}{ Vaginal $\mathrm{pH}$} \\
\hline$<4.5$ & REF & - & - & - & \\
\hline $4.5-5.0$ & 10.33 & 0.03 & 1.13 & 494.62 & \\
\hline$>5.1$ & 12.18 & 0.02 & 1.33 & 583.99 & \\
\hline \multicolumn{6}{|l|}{ Nugent Gram stain score } \\
\hline $0-3$ & REF & - & - & - & \\
\hline $4-6$ & 5.10 & 0.26 & 0.36 & 72.00 & \\
\hline $7-10$ & 9.53 & 0.00 & 1.77 & 95.71 & \\
\hline \multicolumn{6}{|l|}{ Age } \\
\hline $40-45$ & REF & - & - & - & \\
\hline $29-39$ & 0.22 & 0.11 & 0.03 & 1.36 & \\
\hline $18-28$ & 0.30 & 0.20 & 0.05 & 1.69 & \\
\hline $12-17^{\dagger}$ & - & - & - & - & \\
\hline \multicolumn{6}{|l|}{ Ethnicity } \\
\hline Asian & REF & - & - & - & \\
\hline Caucasian $^{\dagger}$ & - & - & - & - & \\
\hline African American & 10.13 & 0.01 & 1.39 & 447.72 & \\
\hline Hispanic $^{\dagger}$ & - & - & - & - & \\
\hline \multicolumn{6}{|c|}{ * OR, odds ratio, estimated using exact logistic regression. } \\
\hline
\end{tabular}

